\title{
Quadrupolar Transitions Evidenced by Resonant Auger Spectroscopy
}

\author{
J. Danger, ${ }^{1,2,3}$ P. Le Fèvre, ${ }^{1}$ H. Magnan, ${ }^{1,2}$ D. Chandesris, ${ }^{1}$ S. Bourgeois,${ }^{4}$ J. Jupille, ${ }^{5}$ T. Eickhoff, ${ }^{6}$ and W. Drube ${ }^{7}$ \\ ${ }^{1}$ Laboratoire pour l'Utilisation du Rayonnement Electromagnétique, Université Paris Sud, \\ Bâtiment 209d, BP 34, 91898 Orsay Cedex, France \\ ${ }^{2}$ Service de Physique et de Chimie des Surfaces et des Interfaces, Commissariat à l'Energie Atomique, \\ 91191 Gif-sur-Yvette Cedex, France \\ ${ }^{3}$ Institut de Physique et de Chimie des Matériaux de Strasbourg, 23 rue du Loess, 67037 Strasbourg, France \\ ${ }^{4}$ Laboratoire de Recherches sur la Réactivité des Solides, Université de Bourgogne, UFR Sciences et Techniques, \\ 9 avenue Alain Savary, BP 47870, 21078 Dijon Cedex, France \\ ${ }^{5}$ Laboratoire CNRS-Saint Gobain "Surface du Verre et Interfaces,” 39 quai Lucien Lefranc, 93303 Aubervilliers, France \\ ${ }^{6}$ Institut für Experimentalphysik, Universität Hamburg, Luruper Chaussee 149, 22761 Hamburg, Germany \\ ${ }^{7}$ Hamburger Synchrotronstrahlungslabor HASYLAB, Deutsches Elektronen-Synchrotron DESY, \\ Notkestraße 85, 22603 Hamburg, Germany \\ (Received 25 June 2001; published 29 May 2002)
}

\begin{abstract}
From absorption spectra, the only way to bring to the fore the occurrence of quadrupolar transitions is to study their angular dependence. Resonant spectroscopies offer a new opportunity to obtain more insight into excited electronic states by studying lineshape and intensity of decay processes. We show here that resonantly excited $\mathrm{Ti} K L_{2,3} L_{2,3}$ Auger spectra of $\mathrm{TiO}_{2}(110)$ carry a clear signature of quadrupolar transitions to localized $e_{g}$ and $t_{2 g} d$-like states, giving access to a direct measurement of crystal field splitting.
\end{abstract}

DOI: $10.1103 /$ PhysRevLett.88.243001

Since it obeys strict selection rules, x-ray absorption (XAS) is an element and orbital-selective probe of both crystallographic and electronic structures of materials. Beyond the dominant features of the absorption spectra arising from excitations which can be interpreted within the dipolar approximation, the absorption pre-edge and nearedge features commonly involve second order quadrupolar transitions (QT) [1]. Although these are of weak intensity, their corresponding terms in the interaction Hamiltonian often contain valuable information. For instance, the magnetism of selected orbitals can be probed via the analysis of QT by x-ray magnetic circular dichroism, as at the $L_{2,3}$ edges of rare earths. At these edges, QT from the $2 p$ levels towards the $4 f$ orbitals (which generally carry most of the magnetic moment in rare earth compounds) give a signal of the same order of magnitude as that due to the dipolar $2 p \rightarrow 5 d$ transitions [2]. In resonant $\mathrm{x}$-ray magnetic scattering, the quadrupolar component $(2 p \rightarrow 4 f)$ dominates the $\mathrm{Sm} L_{3}$-edge and allows a description of the $4 f$ magnetism [3]. Pre- $K$-edge features are often observed in the case of $3 d$ compounds. Associated with transitions to $p$ and $d$ orbitals, they involve both dipolar and quadrupolar transitions and are also important in magnetic measurements [4]. The necessity to take quadrupolar contributions into account is not specific to absorption studies. For a proper determination of surface structures by $\mathrm{x}$-ray standing waves using photoemission detection, nondipolar terms also have to be considered [5]. A picture of the occurrence of QT is therefore of prime interest to achieve a description of the electronic structure of these compounds and to fully account for their physical properties.

A common way to discriminate between dipolar and quadrupolar transitions is based on the interpretation of
PACS numbers: $32.80 . \mathrm{Hd}, 61.10 . \mathrm{Ht}, 71.20 . \mathrm{Be}$

the angular dependence of the XAS cross section, since the electric dipolar $(\varepsilon \cdot \mathbf{r})$ and quadrupolar $(\varepsilon \cdot \mathbf{r k} \cdot \mathbf{r})$ terms of the interaction Hamiltonian are sensitive either to the polarization direction $\varepsilon$ of the $\mathrm{x}$ rays or to both $\varepsilon$ and the direction of the photon wave vector $\mathbf{k}$ [6]. However, this method suffers from severe drawbacks. It can only be applied to single crystals and, to ensure that the weak quadrupolar contributions can be distinguished from the more intense dipolar components, its use is restricted to the study of pre-edge features well separate from the main absorption threshold. Moreover, it does not discriminate between the different contributions.

Resonant Auger spectroscopy permits one to overcome those limitations. It consists in observing an Auger decay while scanning the photon energy through an absorption edge. Because of the presence of a core photoexcited electron in a localized empty orbital, additional decay lines, so-called spectator lines [7], appear at constant binding energies and at higher kinetic energies than the classical decay. Taking place under the influence of the Coulomb field generated by the excited electron, the resonant decay generates unique fingerprints for distinct excitations, provided they differ in their final state energy. Recently, fluorescence measurements brought to the fore $2 p \rightarrow 4 f$ quadrupolar transitions at the $\mathrm{Gd} L_{3}$ edge [8], thanks to a spectator line due to this additional $4 f$ electron. Auger measurements carry the same information on the nature of the electronic transitions near the absorption edge by scanning the very first empty orbitals above the Fermi level [9]. They can be done in a classical photoemission setup, where electrons are collected with high efficiency and energy resolution. In this Letter, the capability of the method to resolve quadrupolar and dipolar contributions is illustrated 
by solving the long-debated question of the pre- $K$-edge of titanium in rutile $\mathrm{TiO}_{2}[10,11]$ and by discriminating the various components of each of the features of the pre-edge spectrum, in good agreement with theoretical predictions $[11,12]$.

The experiments were performed using the tunable highenergy $\mathrm{x}$-ray photoemission station at the BW2 wiggler beam line of HASYLAB, equipped with $\mathrm{Si}(220)$ crystals monochromator and a Scienta SES-200 analyzer (total energy resolution of $0.6 \mathrm{eV}$ at the Ti $K$-edge). Data were collected on a $\mathrm{TiO}_{2}(110)$ rutile single crystal, prepared according to standard procedures in order to obtain the stoichiometric composition [13]. The angular dependence was studied by rotating the sample around a vertical axis. The electron collection direction is fixed in the horizontal plane at $45^{\circ}$ from the incoming $x$-ray beam.

The Ti $K$-edge XAS spectrum (Fig. 1) exhibits a series of three prepeaks called $A_{1}, A_{2}$, and $A_{3}$. In the following, the photon energy is referenced relative to the maximum of $A_{2}$ whose absolute energy position was measured to be $4968.9 \mathrm{eV}$. $A_{1}$ and $A_{3}$ are thus peaking at -2.75 and $+3.0 \mathrm{eV}$ relative photon energy, respectively. Previ-

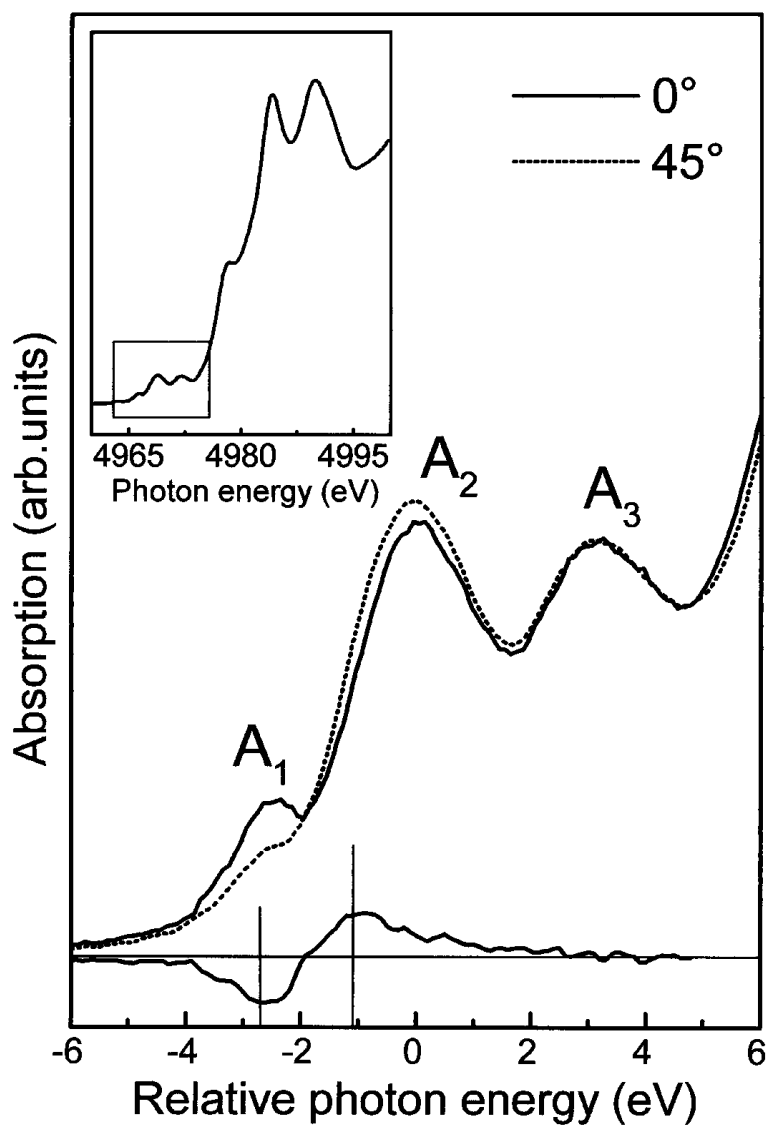

FIG. 1. $A_{1}, A_{2}$, and $A_{3}$ prepeaks of the Ti $K$-absorption edge in rutile. The x-ray wave vector is perpendicular to the $c$ axis and either parallel to $\left(0^{\circ}\right)$ or at $45^{\circ}\left(45^{\circ}\right)$ from the (110) surface normal. The bottom curve is the difference between the two absorption spectra. Inset: absorption threshold at $0^{\circ}$. ous studies used polarization dependent XAS to elucidate the origin of these structures $[10,11]$. Total electron yield spectra were recorded for two different $\mathrm{x}$-ray incidence angles with respect to the (110) surface (Fig. 1). The wave vector of the $\mathrm{x}$ rays is parallel to the (110) surface normal $\left(0^{\circ}, \varepsilon\right.$ at $45^{\circ}$ from the $a$ axis $)$, or at $45^{\circ}$ from it $\left(45^{\circ}, \varepsilon\right.$ parallel to the $a$ axis). At a $K$-edge, due to the highly directional character of the reached $p$ orbitals, the electric dipolar absorption cross section possesses the symmetry of the crystal [1]. It is thus not expected to vary with the $\mathrm{x}$-ray incidence angle (transitions to $p_{x}$ and/or $p_{y}$ orbitals). On the contrary, quadrupolar contributions are expected to have the largest possible intensity variation between $0^{\circ}$ and $45^{\circ}$, since the $d$ symmetry of the probed orbitals confers to their intensity a $\cos (4 \phi)$ dependence [where $\phi$ denotes the rotation of $\varepsilon$ and $k$ in the (100) plane] [1]. All the angular dependences are therefore due to intensity variations of QT. In rutile, the first coordination shell of Ti atoms is composed of six oxygen atoms which form a distorted octahedron [10]. Using the electric quadrupolar Hamiltonian formula, in a basis linked to the octahedron, the only allowed QT in the $0^{\circ}$ geometry are towards $d_{x z}$ and $d_{y z}$ orbitals which belong to the $t_{2 g}$-like representation. In the $45^{\circ}$ geometry, the main transitions allowed are towards the $d_{x^{2}-y^{2}}$ and $d_{z^{2}}$ orbitals, which belong to the $e_{g}$-like representation (transitions towards the $d_{x y} t_{2 g}$-like orbital are also allowed). For the $A_{1}$ prepeak, the maximum intensity at $0^{\circ}$ and the minimum at $45^{\circ}$ are indicative of $t_{2 g}$-like QT. The reversed behavior of the $A_{2}$ prepeak shows that its quadrupolar component is mostly $e_{g}$-like, while the absence of any angular dependence of $A_{3}$ is assigned to a purely dipolar origin. The data meet the previously published results $[10,11]$. However, these did not allow an experimental decomposition of the pre-edge into its various quadrupolar and dipolar contributions. In this goal, an analysis of the Ti $K L L$ resonant Auger lines was undertaken in the pre-edge region of the Ti $K$-edge. Chosen spectra collected in both $45^{\circ}$ and $0^{\circ}$ geometry of the $\mathrm{x}$ rays are shown in Fig. 2. A Au $4 d_{5 / 2}$ photoemission spectrum was recorded before and after each Auger spectrum both for photon energy calibration and Auger spectra normalization. The normal Auger spectrum, recorded for a photon energy well above the ionization threshold, exhibits two pronounced peaks located at $4006.3 \pm 1 \mathrm{eV}$ $\left({ }^{1} D_{2}\right)$ and $3992.9 \pm 1 \mathrm{eV}\left({ }^{1} S_{0}\right)$ kinetic energies. A last structure at $3980 \pm 1 \mathrm{eV}$ may be attributed to $K M$ doubly excited states [14]. As the intensity of these peaks shows the same behavior with the photon energy, we will concentrate in the following on the ${ }^{1} D_{2}$ peak which is the most intense. The series of resonant ${ }^{1} D_{2}$ Auger spectra collected at $0^{\circ}$ and $45^{\circ}$ exhibit three components, labeled $Q_{1}, Q_{2}$, and $D$ (Fig. 2) whose intensities strongly vary with the photon energy. Both $Q_{1}$ and $Q_{2}$ always appear at constant binding energy (959.1 and $961.1 \mathrm{eV}$, respectively), while $D$ has a different behavior. It first appears at a constant binding energy of $963.2 \mathrm{eV}$ (Raman-Auger 


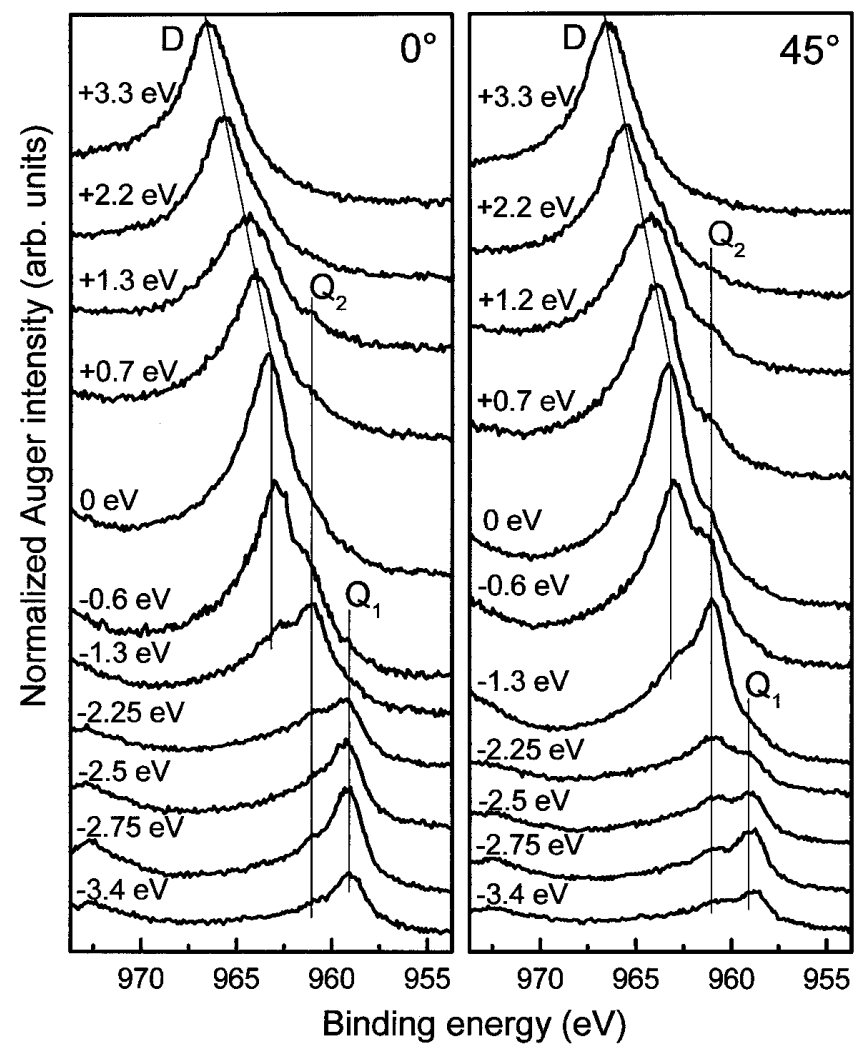

FIG. 2. Ti $K L_{2} L_{3}\left({ }^{1} D_{2}\right)$ Auger line for the indicated different photon energies recorded in the $0^{\circ}$ (left panel) and the $45^{\circ}$ (right panel) geometries.

behavior), until it reaches the position of the ${ }^{1} D_{2}$ Auger peak at a constant kinetic energy of $4006.3 \mathrm{eV}$. In the following, the origin of these different Auger structures and the variations of their intensity both with photon energy and x-ray incidence angle will be discussed.

To examine the intensity of the $Q_{1}, Q_{2}$, and $D$ peaks, the Auger spectra were assumed to be a simple sum of these three components using Lorentzian line shapes and an integral background. The results are presented in Fig. 3 . $Q_{1}$ has a maximum intensity on the maximum of the $A_{1}$ prepeak $(-2.75 \mathrm{eV})$ and follows the same angular dependence. Considering both resonant behavior and angular dependence, $Q_{1}$ is assigned to an Auger decay following the quadrupolar excitation of a $1 s$ electron in the $t_{2 g}$-like orbitals. Moreover, since it appears at constant binding energy, it can be identified as a spectator line. The intensity of $Q_{2}$ shows a maximum just below $A_{2}(-1.0 \mathrm{eV})$ roughly where the difference between the two XAS spectra recorded at $0^{\circ}$ and $45^{\circ}$ is maximum (Fig. 1). Unlike for $Q_{1}$, it increases from $0^{\circ}$ to $45^{\circ}$. $Q_{2}$ can therefore be attributed to a spectator line due to an additional valence electron promoted in the $e_{g}$-like orbitals via a QT. Last, the $D$ peak is attributed to decay following a dipolar transition of the $1 s$ electron towards the $p$ empty states of continuum, since it finally stabilizes at the position of the classical Auger line. Consistently, its intensity does not

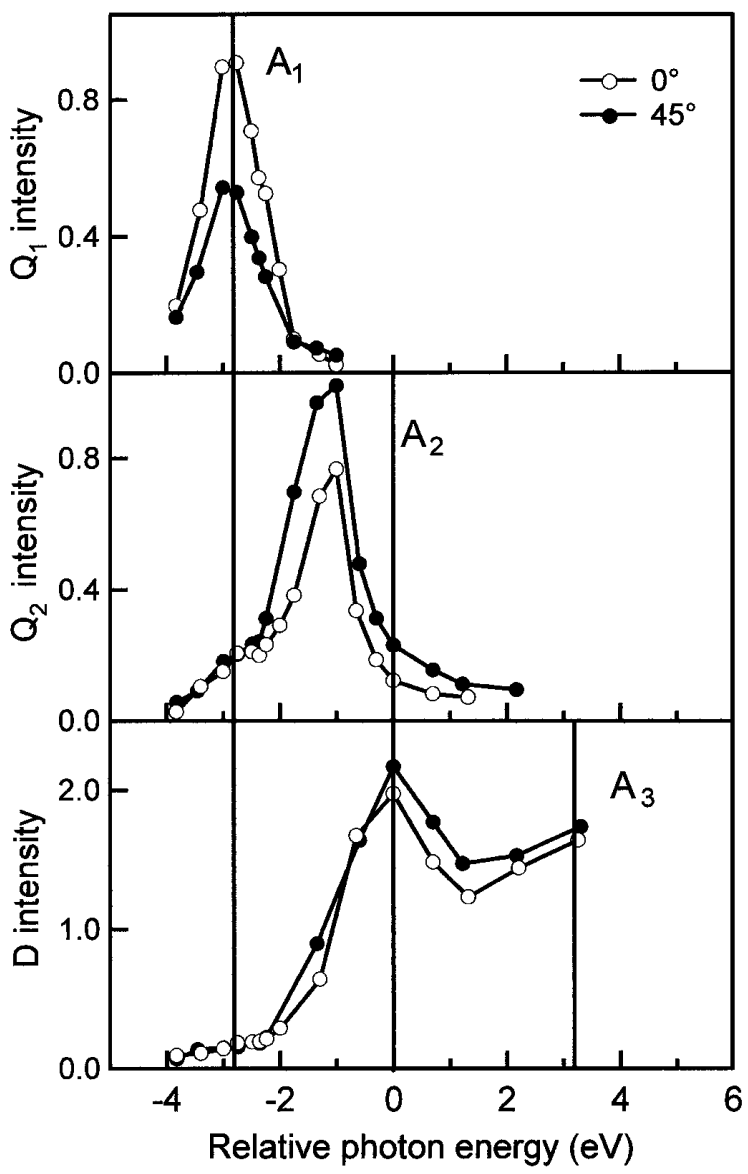

FIG. 3. Intensities of the three peaks $Q_{1}$ (top), $Q_{2}$ (middle), and $D$ (bottom) of the resonant Ti $K L_{2} L_{3}\left({ }^{1} D_{2}\right)$ Auger structure in the $0^{\circ}$ (open circles) and $45^{\circ}$ (closed circles) geometries. The vertical lines indicate the position of the $A_{1}, A_{2}$, and $A_{3}$ absorption prepeaks.

show any significant angular variation (bottom of Fig. 3). Very weak (almost zero) in the $A_{1}$ region, it is roughly twice that of $Q_{2}$ in the $A_{2}$ region. Assuming equal Auger decay rates for the different intermediate states, this intensity analysis of the different Auger components gives an estimate for the relative contributions of quadrupolar and dipolar transitions in the prepeaks. Thus, $A_{1}$ has a purely quadrupolar origin, $A_{3}$ is purely dipolar, and dipolar transitions have equal contributions in $A_{2}$ and $A_{3}$; a quadrupolar contribution to $A_{2}$ is detected on its low-photon-energy side. All these results are in agreement with the calculations presented in Ref. [11].

The origin of the three components $Q_{1}, Q_{2}$, and $D$ of the resonant Auger spectra can also be determined by considering their relative binding energy. Related to the same $K L L$ Auger decay in the presence of a spectator electron, each of those decays differs only by the nature of the orbital in which the electron has been excited. The screening of the core holes by an electron located in a $3 d$ orbital, which is more localized than $p$ continuum states, is expected to lower the final energy of the titanium ion; spectator lines 
associated with $Q_{1}$ and $Q_{2}$ thus show up at binding energies lower than the Raman Auger line $D$. Moreover, in a simple picture of the Raman regime where one considers first a virtual transition of the $1 s$ photoelectron towards a bound orbital, the energy of the decay process is lowered by the binding energy of this bound level [15]. Since the $t_{2 g}$ orbitals have a higher binding energy than the $e_{g}$ orbital, an Auger decay after excitation to $t_{2 g}$ levels appears at a lower binding energy than after excitation to $e_{g}$ levels. At first order, the kinetic energy difference $(2.0 \mathrm{eV})$ between $Q_{1}$ and $Q_{2}$ measures this energy splitting due to crystal field effects. Another precise measurement of crystal field splitting is given by the photon energy difference between the resonance positions of $Q_{1}$ and $Q_{2}(1.75 \mathrm{eV})$. These two measured values are a bit different. This can be explained by the screening effects in the Auger final state which slightly differs for an electron in the $t_{2 g}$ orbitals ( $\pi$ bond with the $\mathrm{O}$ neighbors) or in the more delocalized $e_{g}$ orbitals ( $\sigma$ bond) [16]. Therefore the kinetic energies of $Q_{1}$ and $Q_{2}$ allow the assignment of these features to Auger decays in the presence of the spectator electron into $t_{2 g}$-like and $e_{g}$-like orbitals, respectively, in full agreement with the angular analysis which is therefore not mandatory for this identification.

In the region of the $\mathrm{Ti} K$-edge in $\mathrm{TiO}_{2}$, the resonant behavior of the $K L L$ Auger lines has been used to unravel the different contributions to the absorption spectra. Raman Auger lines following dipolar excitation towards $p$ states, and spectator lines following QT towards $e_{g}$ and $t_{2 g}$ states, split by fine crystal field effects, are clearly discriminated. These decays are unambiguously identified by analyzing either their binding energy or their resonant energy, and an estimate of the $t_{2 g}-e_{g}$ splitting is directly obtained. Therefore, resonant Auger spectra offer direct and separate fingerprints for the different transitions occurring in the studied absorption edge, either to the continuum or to bound states, such as, e.g., quadrupolar transitions to $d$ states at $K$-edges. Spectator lines can be phenomenologically explained and attributed. Here, the quadrupolar origin of the two observed spectator lines has been clearly evidenced. As an experimental method to bring QT to the fore, resonant Auger is certainly more efficient than angular dependent XAS, which allows one to vary only the relative weights of dipole and quadrupole contributions in the nonselective mixture of the absorption signal. These two contributions are clearly separated in the Auger spec- tra, so that their energy position and intensity allow an identification of the QT not only in resolved absorption prepeaks. Next, angular XAS can be performed only on single crystals, whereas resonant Auger measurements can be carried out on powders or amorphous materials. In this case, the angular analysis would be pointless (left and right sides of Fig. 2 would be the same), but same Raman and spectator lines will be observed since they are purely due to the local electronic structure. Angular XAS relies on the orientation of the electronic orbitals with respect to the axes of the infinite and periodic crystallographic structure, whereas resonant Auger measurement is a real selective and local probe of the near-Fermi-level empty orbitals, provided they are localized enough. Last, the sensitivity of electron spectroscopy to the first atomic planes also opens new prospects to show the differences of the electronic structure near the surface.

This work was supported by the E.U. program for access to large facilities. We thank D. Cabaret, Y. Joly, P. Sainctavit, C. Brouder, and M. A. Arrio for instructive discussions, and H. Schulte-Schrepping and J. Heuer for implementing the $\mathrm{Si}(220)$ monochromator.

[1] C. Brouder, J. Phys. Condens. Matter 2, 701 (1990).

[2] F. Baudelet et al., J. Electron Spectrosc. Relat. Phenom. 62, 153 (1993); H. Matsuyama et al., J. Electron Spectrosc. Relat. Phenom. 92, 31 (1998).

[3] K. Dumesnil et al., J. Phys. Condens. Matter 12, 3091 (2000).

[4] W. Neubeck et al., Phys. Rev. B 60, R9912 (1999).

[5] G. J. Jackson et al., Phys. Rev. Lett. 84, 2346 (2000).

[6] J. E. Hahn et al., Chem. Phys. Lett. 88, 595 (1982); G. Dräger et al., Phys. Status Solidi (b) 146, 287 (1988).

[7] G. S. Brown et al., Phys. Rev. Lett. 45, 1937 (1980).

[8] M. Krisch et al., Phys. Rev. Lett. 74, 4931 (1995).

[9] W. Drube, R. Treusch, and G. Materlik, Phys. Rev. Lett. 74, 42 (1995).

[10] B. Poumellec et al., Phys. Status Solidi (b) 164, 319 (1991).

[11] T. Uozumi et al., Europhys. Lett. 18, 85 (1992).

[12] Y. Joly et al., Phys. Rev. Lett. 82, 2398 (1999).

[13] S. Petigny et al., Surf. Sci. 410, 250 (1998).

[14] T. LeBrun et al., Phys. Rev. A 60, 4667 (1999).

[15] T. Åberg and B. Crasemann, in Resonant Anomalous $X$-Ray Scattering, edited by G. Materlik, C. J. Sparks, and K. Fischer (North-Holland, Amsterdam, 1994), p. 430.

[16] P. I. Sorantin and K. Schwarz, Inorg. Chem. 31, 567 (1992). 